HNO 2020 $68: 59-68$

https://doi.org/10.1007/s00106-019-00801-3

○ Der/die Autor(en) 2019

Wissenschaftliche Leitung

M. Canis, München

T. Hoffmann, Ulm

J. Löhler, Bad Bramstedt

P. Mir-Salim, Berlin

S. Strieth, Mainz

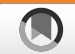

CME

\section{Zertifizierte Fortbildung}

\section{Angiolytische Laserverfahren am Kehlkopf}

\section{Phonochirurgische Aspekte bei transoraler Lasermikrochirurgie}

\author{
S. Strieth ${ }^{1} \cdot$ J. Hagemann ${ }^{1} \cdot$ M. Hess ${ }^{2}$ \\ ${ }^{1}$ Hals-Nasen-Ohren-Klinik, Universitätsmedizin Mainz, Mainz, Deutschland \\ ${ }^{2}$ Deutsche Stimmklinik, Hamburg, Deutschland
}

\section{Zusammenfassung}

Online teilnehmen unter:

www.springermedizin.de/cme

Für diese Fortbildungseinheit

werden 3 Punkte vergeben.

\section{Kontakt}

Springer Medizin Kundenservice

Tel. 08007780777

(kostenfrei in Deutschland)

E-Mail:

kundenservice@springermedizin.de

\section{Informationen}

zur Teilnahme und Zertifizierung finden Sie im CME-Fragebogen am Ende des Beitrags.
Durch transorale Lasermikrochirurgie wurden bei der Larynxkarzinomtherapie temporäre Tracheotomien reduziert, die Organerhaltraten erhöht und funktionelle Resultate verbessert. Goldstandard für die laserbasierte transorale Resektion eines Larynxkarzinoms ist der Einsatz des $\mathrm{CO}_{2}$-Laser, dabei sind onkologische Radikalität und postoperative Stimmfunktion individuell abzuwägen. Angiolytische Lasereffekte ermöglichen eine Beeinflussung des Tumormikromilieus durch zielgerichtete Gefäßobliteration und Blockierung der Angiogenese mit Schonung von schwingungsfähigem Gewebe für eine gute Stimmfunktion. Mit der Verabschiedung der S3-Leitlinie zur Diagnostik, Therapie und Nachsorge des Larynxkarzinoms wird eine nationale evidenzbasierte Standardisierung gefördert. International nimmt die Evidenz zur Kalium-Titanyl-Phosphat-Laser-Therapie laryngealer Schleimhautdysplasien und von T1a-Larynxkarzinomen zu. Auch bei juveniler Papillomatose und der Stützlaryngoskopie unter Narkose sowie für ausgewählte Patienten in Lokalanästhesie werden angiolytische Laser eingesetzt.

\section{Schlüsselwörter}

Larynxkarzinom $\cdot$ KTP $\cdot$ TLM $\cdot$ Larynxpapillomatose $\cdot$ Stimme

\section{Lernziele}

\section{Nach der Lektüre dieses Beitrags ...}

- kennen Sie den Stellenwert von $\mathrm{CO}_{2}$ - und Kalium-Titanyl-Phosphat(KTP)-LaserAnwendungen vor dem Hintergrund der aktuellen Larynxkarzinomleitlinie,

- können Sie individualisierte Therapieentscheidungen durch die Wahl des Laserverfahrens für die transorale Lasermikrochirurgie (TLM) treffen,

- sind Sie informiert über den gegenwärtigen Stand eines evidenzbasierten Einsatzes von angiolytischen Lasern im Rahmen der Stützlaryngoskopie und in Lokalanästhesie. 


\section{Hintergrund}

Bei der chirurgischen Behandlung benigner und maligner Schleimhautveränderungen des oberen Aerodigestivtrakts haben Laserverfahren einen hohen Stellenwert. Ausgehend von den Arbeiten von Jako [1] und Steiner [2] haben sie insbesondere am Kehlkopf die klinische Praxis in den vergangenen Jahrzehnten revolutioniert, indem der Organerhalt ganz wesentlich verbessert werden konnte. Obwohl die funktionellen Stimmresultate recht gut sind, kommt es immer noch zu Verlusten an Stimmqualität [3]. Angesichts der hohen Bedeutung der Stimme im soziokulturellen und professionellen Kontext müssen sich aktuelle Weiterentwicklungen im Bereich der Lasertechnologie am Larynx der Herausforderung stellen, ein bestmögliches Stimmresultat zu erzielen.

Zur Beherrschung benigner Erkrankungen und ausgewählter Frühformen bösartiger Prozesse etablieren sich daher gegenwärtig zielgerichtete Laserverfahren zunehmend als Alternative zu bisherigen thermisch tief einschneidenden Techniken.

Folkman (1933-2008) postulierte 1971, dass das Wachstum von Tumoren über einige Millimeter hinaus und die Erlangung von Metastasierungspotenzial davon abhinge, ob ein funktionierendes Tumorblutgefäßsystem gebildet werden könne (Angiogenese) [4]. Die Antiangiogenese wurde in den letzten Jahrzehnten als effektives medikamentöses Therapiekonzept auch klinisch etabliert [5]. Zeitels et al. publizierten 2008 [4] erstmals das Konzept einer laserbasierten gezielt antivaskulären Therapieform am Larynx [6]. Hierbei ist bei der Mikrolaryngoskopie bewusst auf torquierte Gefäßneubildungen mit chaotischer Gefäßarchitektur - „intraepithelial capillary papillary loops" (ICPL) - zu achten, die auch schon Jako und Kleinsasser 1966 beschrieben. Durch zielgerichtete Laserobliteration der angiogenetischen Gefäße wird das Tumormikromilieu verändert - die Tumorzelle selbst wird dadurch zunächst nur indirekt zum Ziel. Sekundär kommen allerdings auch hier thermische Effekte auf das Epithel und damit auf die Tumorzelle zum Tragen. Aktuell nimmt die Evidenzlage zur Verwendung angiolytischer Laser erheblich zu $[6,7,8,9,10]$. Dies führte bereits $2014 \mathrm{zu}$ einer Stellungnahme der US-amerikanischen otorhinolaryngologischen Fachgesellschaft, die zur klinischen Implementierung dieser photoangiolytischen Verfahren ermutigt [11]. Vor dem Hintergrund der Veröffentlichung der ersten AWMF-S3-Leitlinie zu Diagnostik, Therapie und Nachsorge des Larynxkarzinoms erscheint es geboten, sich auch in Deutschland mit diesen innovativen Entwicklungen auseinanderzusetzen. In der neuen Version der Leitlinie wurde dementsprechend auch der allgemeinere Terminus "Laser" verwendet und damit bewusst die Beschränkung auf den bisher bewährten $\mathrm{CO}_{2}$-Laser vermieden.

\footnotetext{
> Merke
}

In der aktuellen S3-Leitlinie wird bewusst eine Einschränkung auf den bisher bewährten $\mathrm{CO}_{2}$-Laser vermieden.

\section{Transorale Laserchirurgie}

Lange Zeit standen zur chirurgischen Behandlung von Erkrankungen des Kehlkopfs nur offene chirurgische Verfahren zur Verfü-
Angiolytic laser applications for the larynx. Phonosurgical concepts for transoral laser microsurgery

Transoral laser microsurgery (TLM) for treatment of laryngeal cancer has reduced temporary tracheotomies, increased organ preservation rates, and improved functional results. Gold standard for laser-based transoral resection of laryngeal cancer is the application of $\mathrm{CO}_{2}$ lasers. Oncologically safe radical resection and postoperative voice outcome must be weighed up individually. Angiolytic laser effects enable modification of the tumor micromilieu by targeted obliteration of microvessels and antagonization of angiogenesis with preservation of vibrating laryngeal tissue for good voice function. Introduction of the German S3 guideline on diagnosis, treatment, and follow-up of laryngeal cancer is a critical step towards national evidence-based standardization. Internationally, the evidence for treatment of laryngeal mucosal dysplasia and T1a cancer with angiolytic potassium titanyl phosphate (KTP) lasers is increasing. Angiolytic lasers are also used for juvenile papillomatosis and suspension microlaryngoscopy under general anesthesia or local anesthesia in selected patients.

\section{Keywords}

Laryngeal cancer · KTP · TLM · Laryngeal papillomatosis · Voice

gung. Erst in der Nachkriegszeit wurde die damals als Standard angesehene Laryngofissur um transorale Verfahren mittels Stützlaryngoskopie erweitert [12]. Zunächst war auch dabei die Therapie auf nichtneoplastische Kehlkopferkrankungen beschränkt - z.B. der beidseitigen Rekurrensparese. Transorale Verfahren wurden aber relativ schnell auf die Behandlung von malignen Tumoren ausgeweitet. Bei der cold steel-Resektion bestand jedoch in der unmittelbaren Nähe zum Atemweg eine besondere Gefahr von Aspirationsereignissen durch Blutungen während oder nach der Operation. Eine präventive oder notfallmäßige Tracheotomie im Zuge der Behandlung kam vor. Nicht zuletzt auch aus Besorgnis um das postoperative Stimmresultat wurde daher zur Schonung der laryngealen framework-Strukturen, d.h. des Larynxskeletts, nach neuen chirurgischen Methoden gesucht und die transorale Lasermikrochirurgie (TLM) entwickelt $[2,13]$.

In der aktuellen S3-Leitlinie zu Diagnostik, Therapie und Nachsorge des Larynxkarzinoms stellt die TLM eine wichtige therapeutische Modalität dar [14]. Insbesondere glottische T1- und T2Tumoren werden in den meisten Zentren derzeit laserchirurgisch angegangen, sofern dies in einer präinterventionellen Panendoskopie funktionell und onkologisch vertretbar erscheint. Während bei größeren Tumoren (T2, ausgesuchte T3-Fälle) der Einsatz des Kohlenstoffdioxid $\left(\mathrm{CO}_{2}\right)$-Lasers den Goldstandard darstellt, kommen zur Beherrschung von dysplastischen Vorstufen und frühen Formen (T1a) zunehmend auch neuartige, photoangiolytische Laserverfahren zum Einsatz. Diese Verfahren waren lange nicht ausreichend in prospektiven Studien untersucht und sind daher auch zu Recht noch nicht explizit in der aktuell gültigen Larynxkarzinomleitlinie verankert. Sie sind allerdings besonders interessant, weil sie auch phonochirurgischen Ansprüchen gerecht werden können.

Aufgrund der Problematik der thermisch veränderten Absetzungsränder oder Behandlungsareale bleibt weiterhin bei allen transoralen Laserverfahren eine Kontrollmikrolaryngoskopie nach 4-6 Wochen zum histologischen Ausschluss eines Tumor- 
Tab. 1 Chordektomietypen bei transoraler Lasermikrochirurgie mittels $\mathrm{CO}_{2}$-Laser $\left(\mathrm{TLM}^{-} \mathrm{CO}_{2}\right.$ ) nach der European Laryngological Society [17] Chordektomietyp Lokalisation/Ausmaß

\begin{tabular}{|l|l|}
\hline Typ I & Unilateral - subepithelial \\
\hline Typ II & Unilateral - subligamental \\
\hline Typ III & Unilateral - transmuskulär \\
\hline Typ IV & Unilateral - komplett \\
\hline Typ V & Bilateral - erweitert \\
\hline
\end{tabular}

Tab. 2 Klassifikationsvorschlag zur TLM-KTP nach Mallur et al. 2014 [24]

\begin{tabular}{|l|l|}
\hline Klassifikation & Ausmaß \\
\hline KTP V & Kein Kontakt, rein angiolytisch \\
\hline KTP 1 & Kein Kontakt, Epithel intakt \\
\hline KTP 2 & Kein Kontakt, Epithel unterbrochen \\
\hline KTP 3 & $\begin{array}{l}\text { Mit oder ohne Kontakt, Epithelablation ohne } \\
\text { Gewebeabtragung }\end{array}$ \\
\hline KTP 4 & Kontakt, Epithelablation mit Gewebeabtragung \\
\hline
\end{tabular}

TLM-KTP transorale Lasermikrochirurgie mittels Kalium-Titanyl-Phosphat-Laser

residuums und zur Einleitung geeigneter Salvagemaßnahmen empfehlenswert.

\section{Stützmikrolaryngoskopie in Intubationsnarkose}

\section{$\mathrm{CO}_{2}$-Laser}

Beim $\mathrm{CO}_{2}$-Laser handelt es sich um einen schneidenden Laser mit $10.600 \mathrm{~nm}$ Wellenlänge, mit dem das Gewebe punktuell mit hoher Energie thermisch erhitzt und präzise durchschnitten wird. Das Verfahren wird in der Regel mikroskopisch kontrolliert mittels Stützlaryngoskopie in Vollnarkose angewendet. Die Methode wurde weiterentwickelt, insbesondere zu sog. piecemeal-Resektionen: Hierunter versteht man Tumorentfernungen, die Schnittführungen durch den Tumor und die Komplettresektion in Teilen erlauben, um letztendlich den geforderten Sicherheitsabstand von 1-3 mm zu erreichen [15].

Trotz der relativ geringen geforderten Sicherheitsabstände bei Resektionen am Larynx kommt es im Bereich der Stimmlippen zu unterschiedlich großem Verlust funktionell wichtiger Strukturen und Gewebeschichten. Hierbei ist insbesondere die durch Vernarbung hervorgerufene Verringerung der regelrecht verschieblichen und damit schwingungsfähigen Lamina propria bedeutsam, die zu einer Verschlechterung der Stimmbildung führt. Insgesamt kommt es aber zu akzeptablen funktionellen Stimmresultaten bei umschriebenen Resektionen [16]. Allerdings ist die postoperative Stimmfunktion bei der Behandlung von T1a-Larynxkarzinomfrühformen häufig nicht besser als der präoperative Befund [9].

Das Ausmaß der Resektion wurde vor 20 Jahren von der European Laryngological Society (ELS) in 5 Chordektomietypen unterteilt (Tab. 1), um eine Vergleichbarkeit der onkologischen und funktionellen Resultate nach transoraler Lasermikrochirurgie mittels $\mathrm{CO}_{2-}^{-}$ Laser $\left(\mathrm{TLM}-\mathrm{CO}_{2}\right)$ zu ermöglichen [17].
Gegenwärtig ist davon auszugehen, dass funktionelle und onkologische Langzeitergebnisse nach TLM- $\mathrm{CO}_{2}$ und primärer Radiotherapie bei der Therapie glottischer Plattenepithelkarzinomfrühformen (T1a) vergleichbar sind [18, 19].

D Merke

Funktionelle und onkologische Langzeitergebnisse nach $\mathrm{TLM}-\mathrm{CO}_{2}$ und primärer Radiotherapie bei glottischen Plattenepithelkarzinomfrühformen (T1a) sind vergleichbar.

Bemerkenswert ist, dass bei beiden Behandlungskonzepten aber immer noch relevante Beeinträchtigungen in Bezug auf die Stimmfunktion vorkommen [18, 20]. In beiden Fällen wird vielfach eine logopädische Stimmtherapie durchgeführt, obwohl prospektive Studien fehlen und sich auch in retrospektiven Analysen kein signifikanter Vorteil in Eigen- oder Fremdeinschätzung belegen lässt [15].

\section{KTP-Laser}

Das Konzept der Antiangiogenese ist mittlerweile in der Onkologie etabliert, und es kommt bei speziellen Entitäten - z.B. beim Kolonkarzinom - zur zielgerichteten medikamentösen Tumorbehandlung bereits klinisch zum Einsatz [5]. In der Kopf-Hals-Onkologie findet das Konzept in dieser Form nach ernüchternden klinischen Studien mit Vascular-Endothelial-Growth-Factor(VEGF)Antikörpern gegenwärtig noch keine Anwendung.

Mit dem Kalium-Titanyl-Phosphat(KTP)-Laser steht in der Laryngologie ein angioablatives, nicht einschneidendes Laserverfahren zurVerfügung, das das Konzept einer antivaskulären Therapie interventionell-chirurgisch aufgreift. Bei $532 \mathrm{~nm}$-Wellenlänge wird die Energie des Lasers durch Hämoglobin absorbiert, sodass kleinere Blutgefäße selektiv verschlossen werden [21]. Daher ist vonseiten des Laserschutzes auch die relevante Gefahr von möglichen Netzhautschäden durch aberrantes Laserlicht zu berücksichtigen. Der besondere Vorteil des angiolytischen KTP-Verfahrens besteht aber in der konzeptionellen Möglichkeit der Schonung schwingungsfähigen Gewebes in der Lamina propria.

> Merke

Ein besonderer Vorteil der KTP-Anwendung am Kehlkopf besteht in der konzeptionellen Möglichkeit der Schonung schwingungsfähigen Gewebes in der Lamina propria.

Mittlerweile existieren Therapieerfahrungen bei der Larynxpapillomatose [22], bei laryngealen Dysplasien und Frühformen glottischer Karzinome $[6,7,8,9,10]$.

Der KTP-Laser wird zur optimal kontrollierten Anwendung mit einer flexiblen Glasfaser nach Exposition des Larynx mittels Stützlaryngoskopie appliziert (Abb. 1). Die applizierte Energie hängt von der Faserstärke, dem Abstand zum Gewebe und der Pulsrate ab. Diese Tatsache ist für standardisierte Therapieansätze von Bedeutung [23]. Mallur et al. publizierten einen Vorschlag für eine Klassifizierung einer KTP-Laser-Behandlung, die zwischen reiner Angioablation mit kontaktfreier Anwendung des Lasers und einer Gewebeabtragung mit direktem Kontakt der Laserfaser in 5 Gra- 

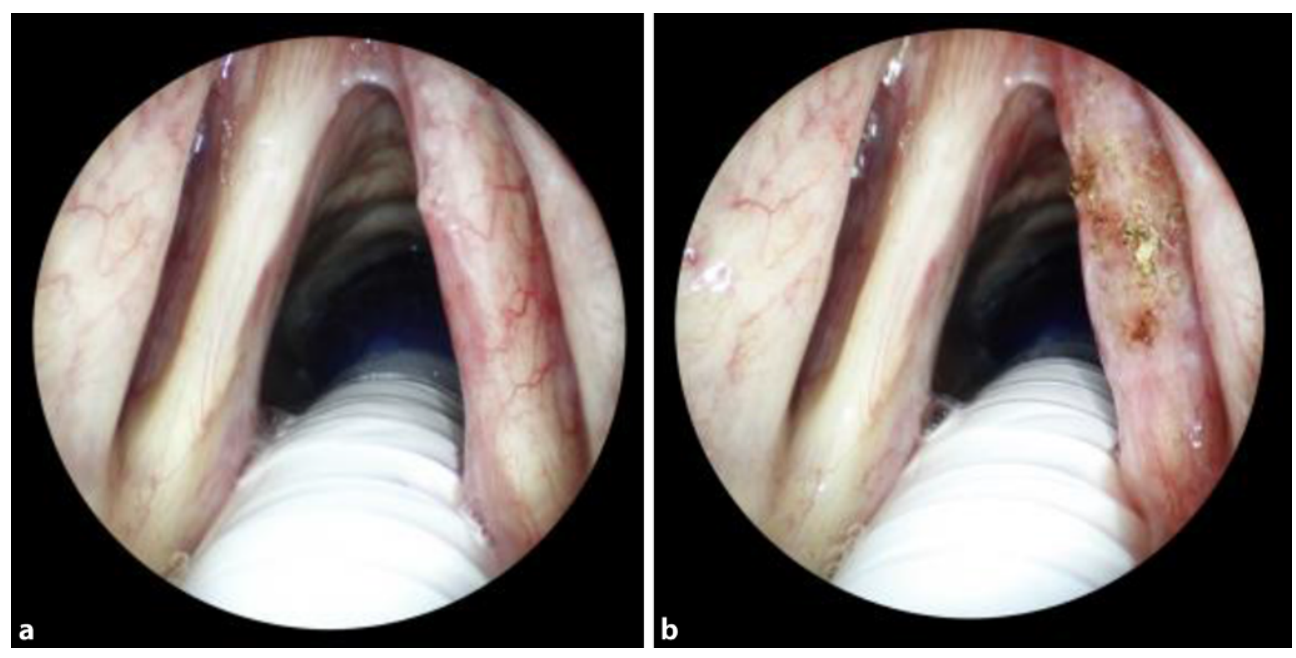

Abb. 1 Stützlaryngoskopische Exposition des Larynx in Narkose zur transoralen Lasermikrochirurgie mittels Kalium-Titanyl-Phosphat-Laser (TLM-KTP). a Auf der rechten Stimmlippe offensichtliche Angiogenese als indirekter Hinweis auf dysplastische Schleimhaut, histologisch bereits invasiv (T1a). b Durch KTP-3-Behandlung nach Mallur sichtbares unmittelbares Verblassen der Gefäße - neben thermischen Effekten
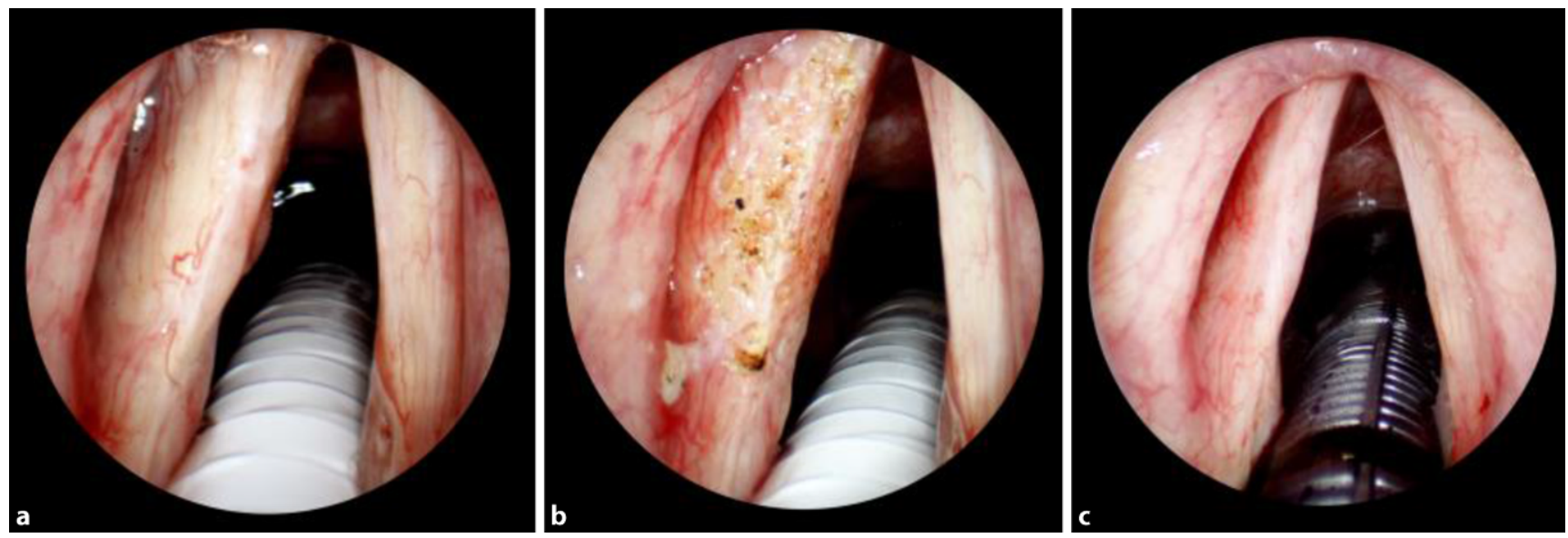

Abb. 2 ム Transorale Lasermikrochirurgie mittels Kalium-Titanyl-Phosphat-Laser (TLM-KTP) eines a einseitigen Carcinoma in situ der linken Stimmlippe. Voice Handicap Index (VHI) initial $52(0<\mathrm{VHI}-30<120)$. b Infolge KTP-3-Behandlung nach Mallur c unauffällige Larynxbefunde ohne torquierte angiogenetische Gefäße bei Kontrollmikrolaryngoskopie nach 6 Monaten. Patient auch 3 Jahre später tumorfrei mit zufriedenstellendem VHI-30-Wert von 19 $(0<\mathrm{VHI}-30<120)$

duierungen (KTP-V, KTP-1, KTP-2, KTP-3 und KTP-4) unterscheidet [24]. Der Vorteil dieser Klassifikation liegt darin, dass die effektive Behandlungstechnik und nicht allein Lasereinstellungsparameter zur Stratifizierung von Kohorten in klinischen Studien verwendet werden könnten (Tab. 2).

Je nach Ausmaß der Behandlung kann die Kontinuität gesunden Epithels und der Lamina propria und damit die Gewebeverschieblichkeit erhalten bleiben oder sich zumindest im Verlauf erfolgreich regenerieren (Abb. 2). Durch höhere Energieapplikation können aber neben subepithelialer Angiolyse auch indirekte thermische Effekte am Epithel induziert werden, sodass es durchaus auch hierbei an der Randkante zu funktionell relevanten Vernarbungen kommen kann.

Eine besondere Stärke der angiolytischen Laser liegt bei der Behandlung großflächiger dysplastischer Schleimhautveränderungen insbesondere dann, wenn diese auf beiden Stimmlippen vorliegen (Abb. 3). In diesen Fällen kann beispielsweise durch eine KTP-V-Anwendung nach Mallur eine Überbehandlung vermieden werden und wertvolle Stimmfunktion erhalten bleiben. Young et al. zeigten in ihrer Studie von 2015 eine signifikante Reduktion der funktionellen Stimmbeschwerden durch KTP-Laser-Behandlung bei Reinke-Ödemen [25]. Der Erhalt der Mikrostruktur des Stimmbildungsorgans oder zumindest die Bewahrung ihrer Regenerationsfähigkeit resultieren in einer verbesserten Stimmfunktion. Dieses wurde in klinischen Studien häufig mithilfe des subjektiven "Stimmhandicaps" durch einen Fragebogen (Voice Handicap Index, VHI, [26, 27]) quantitativ erfasst. Hierbei werden Einschränkungen der Stimmfunktion und Lebensqualität durch die Patienten quantifiziert und in 2 gängigen validen Fragebogeninstrumenten (VHI-10 oder VHI-30) erfasst. Für die Beurteilung des funktionellen Resultats sind grundsätzlich verschiedene Parameter sinnvoll. Am häufigsten wird über Einschätzungen durch die Patienten selbst (z. B. VHI-30) oder durch Untersuchende (GRBAS: Grade/Grad, Roughness/Rauheit, Breathiness/Behauchtheit, Asthenia/Asthenie - „Klangarmut", Strain/Pressen), sowie objektive Messungen mittels Videostroboskopie (z. B. Jitter, Shimmer) in der Literatur berichtet. 

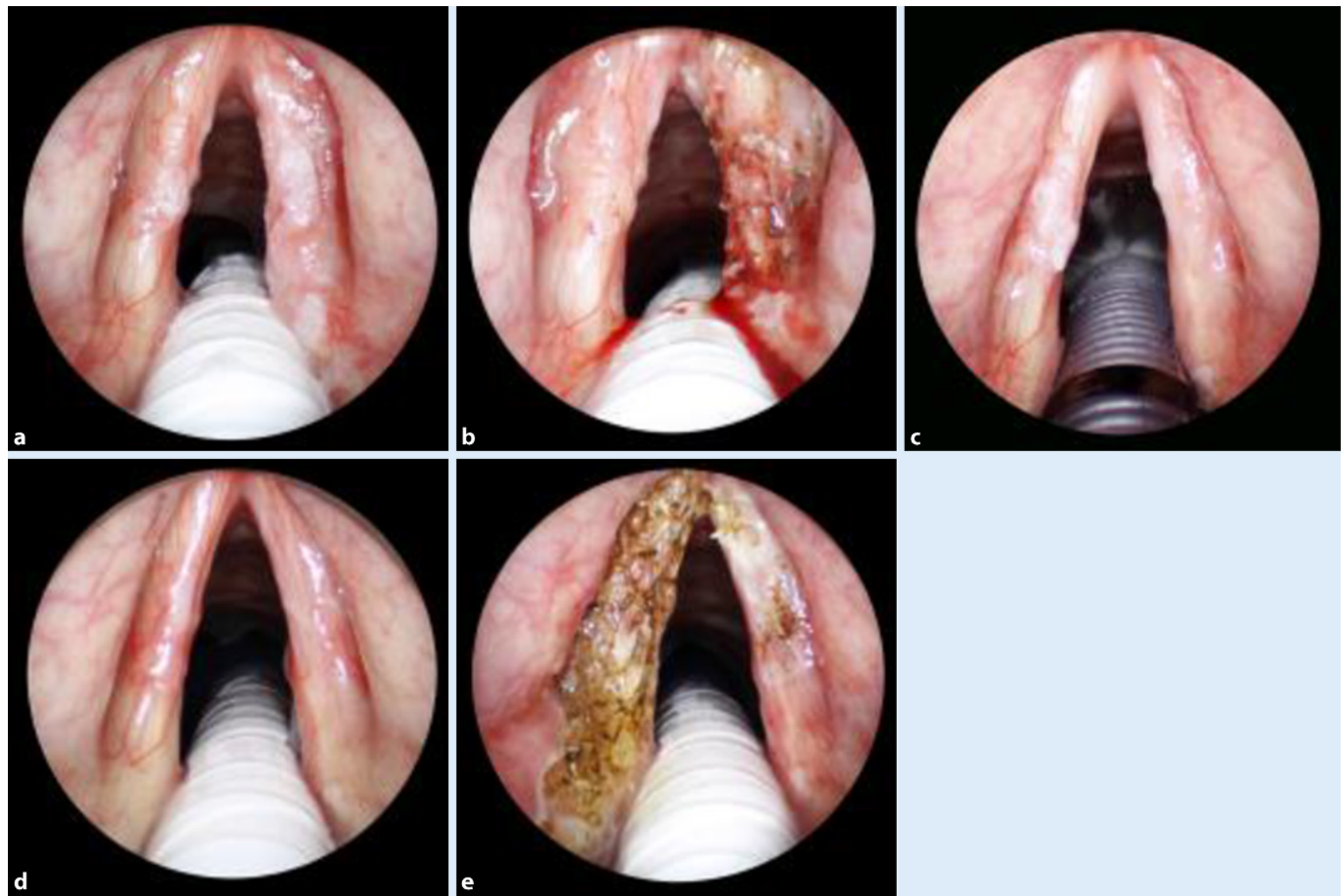

Abb. 3 ॥ Transorale Lasermikrochirurgiemittels Kalium-Titanyl-Phosphat-Laser (TLM-KTP) beider Stimmlippen. a Carcinoma in situ der rechten Stimmlippe und histologisch nicht-invasive hyperkeratotische Schleimhaut der linken Stimmlippe (Voice Handicap Index, VHI-30-Wert = 33;0 $<$ VHI-30 $<120$ ). b Zunächst KTP-4-Laserung nach Mallur rechts und KTP-V nach Mallur links. c Kontrollmikrolaryngoskopie 6 Wochen später mit immer noch großflächig hyperkeratotischer Schleimhaut links, d im weiteren Verlauf persistierend und zunehmend gerötet. e Daher 3 Wochen später operative Revision im Sinne einer KTP-3-Behandlung links und eine erneute kleinfächige Laserung rechts. Danach Epithel unauffällig. Funktionell VHI- $30-W e r t ~ v o n ~ 14(0<\mathrm{VHI}-30<120)$. Patient noch 3 Jahre später dysplasie- und tumorfrei
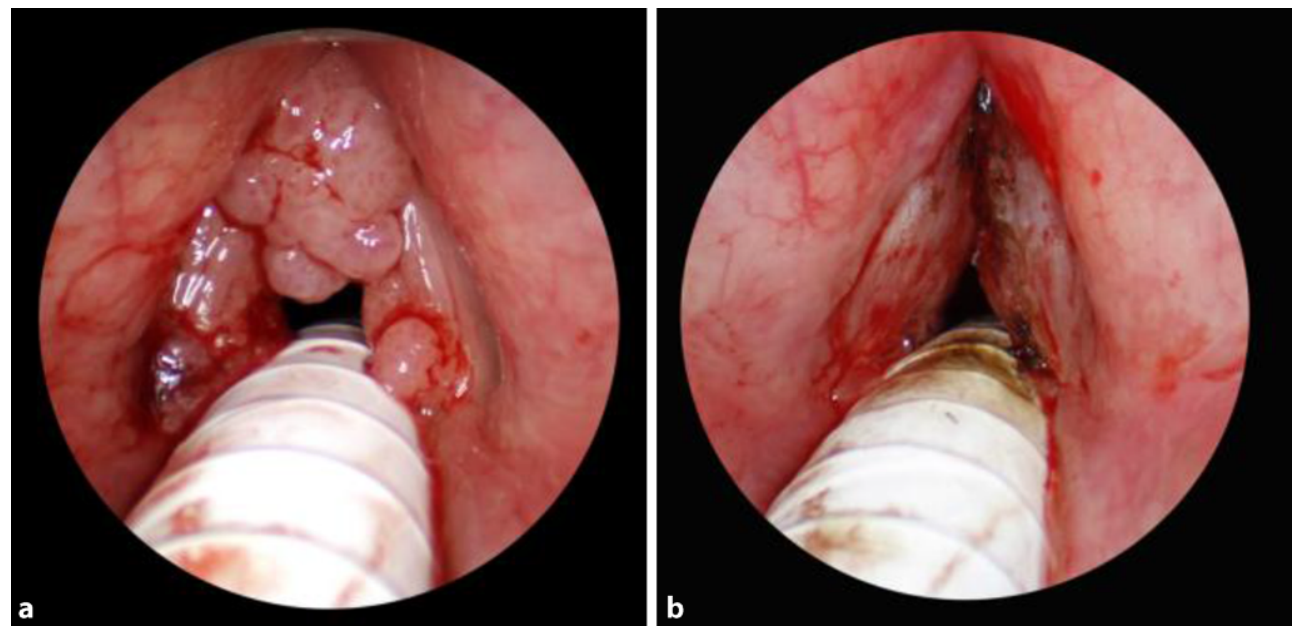

Abb. $4<$ Transorale Lasermikrochirurgiemittels Kalium-TitanylPhosphat-Laser (TLM-KTP) bei juveniler Larynxpapillomatose (a präoperativ; b postoperativ). Trotz KTP-4-Behandlungen der exophytischen Raumforderung weitgehend unbeeinträchtigte Stimmfunktion auch bei wiederholten Eingriffen möglich 

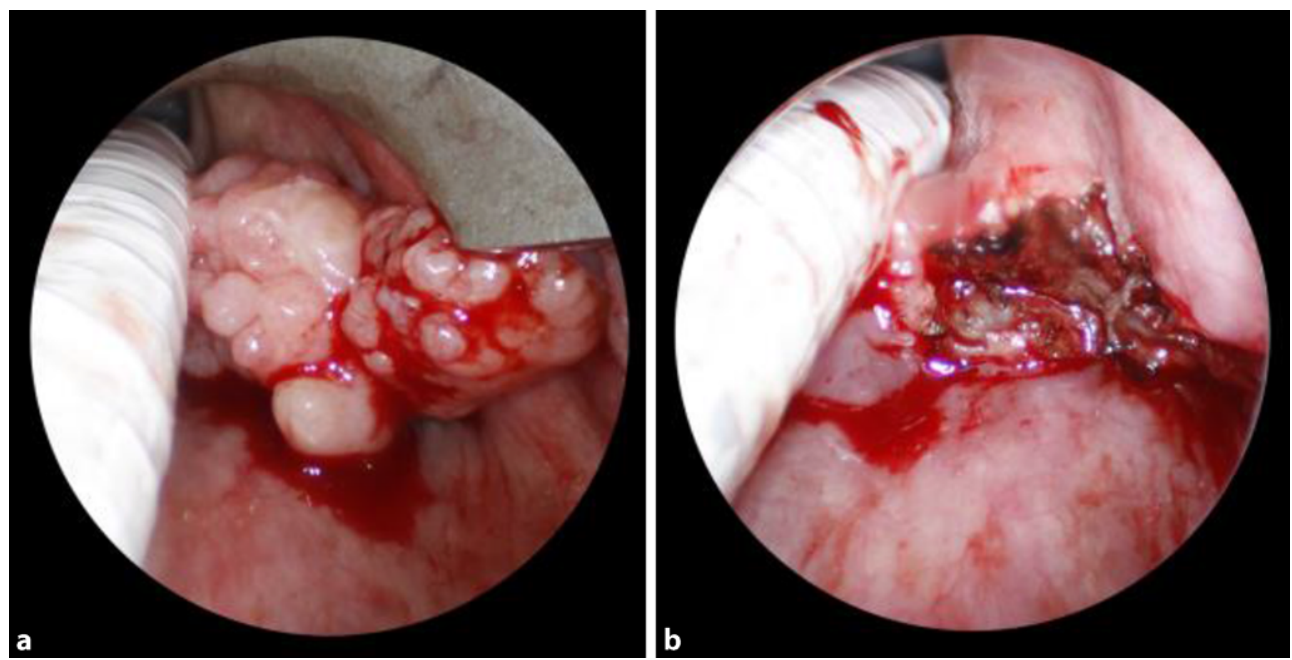

Abb. 54 Transorale Lasermikrochirurgiemittels Kalium-TitanylPhosphat-Laser (TLM-KTP) bei adulter Larynxpapillomatose (a präoperativ; b postoperativ). An funktionell relevanten Lokalisationen, hier Aryregion links, Vorteile durch KTP-Laser (hier KTP-4-Laserung). Engmaschige lupenlaryngoskopische Kontrollen empfohlen

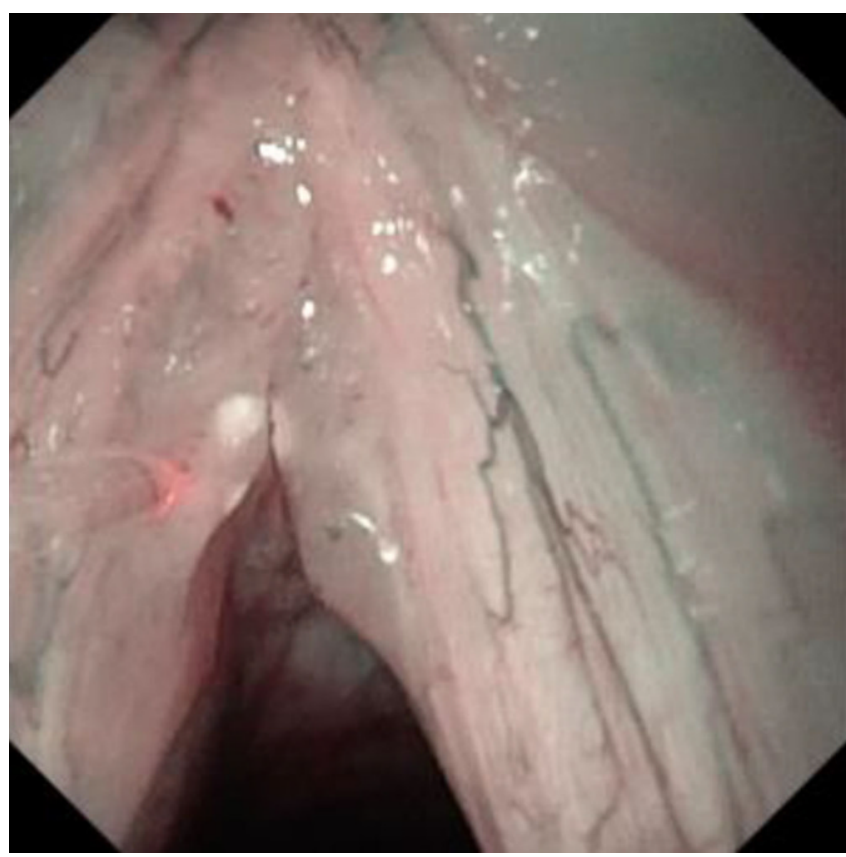

Abb. 6 ム Transnasale "office-based" erfolgte Operation von adulten Larynxpapillomen im Bereich der vorderen Stimmlippenabschnitte. Einführen einer $400 \mu$ m dünnen Glasfaser (links im Bild) durch den Instrumentenkanal des flexiblen Tip-Chip-Kamera-Endoskops. Durch Rotation des Endoskops und Sprühanästhesie mit Lidocain minutenlanges Lasern mit dem photoangiolytischen Laser ohne Sedierung möglich. Durch Blanchieren sichtbarer Koagulationseffekt. Laserpulse bei $4 \mathrm{~W}$ und $30 \mathrm{~ms}$ Pulsdauer mit 200 ms-Pausen vom Patienten nicht spürbar. Unter Narrow-Band-Imaging(NBI)-Spektrallicht noch deutlicher sichtbare Gefäße

$$
\text { - Merke }
$$

Für die Beurteilung des funktionellen Resultats sind grundsätzlich die Einschätzung durch die Patienten selbst oder durch Untersuchende sowie objektive Messungen sinnvoll.

Es ist sinnvoll, solche Parameter auch bei onkologischen Patienten und nicht nur in phonochirurgischen Fällen standardmäßig postoperativ zu erfassen.
In einer prospektiven randomisierten einfach verblindeten Studie wurde nun erstmals VHI-basiert eine verbesserte Stimmfunktion nach transoraler Lasermikrochirurgie mittels Kalium-TitanylPhosphat-Laser (TLM-KTP) im Vergleich zu TLM-CO 2 bei der transoralen Therapie von Larynxkarzinomfrühformen (CIS, T1a) nachgewiesen [9]. Darüber hinaus war die Stimmfunktion postoperativ nach KTP-Anwendung in den meisten Fällen besser als präoperativ, sodass in vielen Fällen kein subjektives Stimmdefizit (VHI-30-Werte $<20$ ) beklagt wurde [9]. Die onkologisch relevante lokale 3-Jahres-Kontrollrate nach TLM-KTP als sekundärer Zielparameter war unter Berücksichtigung umfassender Übersichtsarbeiten in dieser Studie vergleichbar mit anderen etablierten Therapieverfahren auch der Strahlentherapie [28].

Daher lägen gegenwärtig randomisierte Multizenterstudien mit 3- oder 5-Jahres-Kontrollraten als primären Endpunkten nahe. Methodisch wäre bei Therapie in Intubationsnarkose eine einfache Verblindung leicht zu realisieren. Zudem wäre eine Stratifizierung nach Mallur äußerst sinnvoll.

Bei der therapeutischen Abwägung des anzuwendenden Laserverfahrens wiegen stimmfunktionelle Defizite bei der Therapie gutartiger Neubildungen naturgemäß schwerer als bei der Behandlung maligner Tumoren. Dies betrifft in besonderer Weise die Behandlung der juvenilen Larynxpapillomatose, die noch nicht als obligate Präkanzerose gelten darf und die häufig multiple Laserbehandlungen erforderlich macht. Eine Tracheotomie ist grundsätzlich zu vermeiden.

$>$ Cave!

Eine Tracheotomie ist bei der Larynxpapillomatose grundsätzlich zu vermeiden.

Bei der Behandlung der juvenilen Larynxpapillomatose mit dem KTP-Laser existieren daher erste prospektive klinische Studien [29]. Die Strukturschonung erscheint gerade bei dieser Indikation als bedeutsamer Vorteil der Anwendung eines photoangiolytischen Lasers (Abb. 4). Trotz KTP-4-Behandlungen exophytischer Raumforderungen kann auch bei wiederholten Eingriffen die Stimmfunktion weitgehend unbeeinträchtigt bleiben. 
$\checkmark$ Merke

Eine vielversprechende Anwendung des KTP-Lasers erscheint die Therapie der juvenilen Larynxpapillomatose.

Prospektive Langzeituntersuchungen zur Behandlung der adulten Larynxpapillomatose stehen gegenwärtig noch aus, sodass bei Verwendung eines KTP-Lasers zumindest engmaschige Kontrollen erforderlich erscheinen (Abb. 5).

Die TLM-KTP wurde darüber hinaus zur Vermeidung von Rezidiven einer Larynxpapillomatose mit subläsionaler Applikation von Bevacizumab, einem VEGF-Antikörper, kombiniert [30]. Erste prospektive Untersuchungen bei der Larynxpapillomatose deuten darauf hin, dass die medikamentöse Zusatzbehandlung eine sinnvolle Ergänzung der KTP-Laserung darstellen könnte [31].

\section{Flexible Endoskopie in Lokalanästhesie}

Ambulante Verfahren sind mittels flexibler Endoskopie in Lokalanästhesie möglich. Die Therapie von gutartigen und dysplastischen Läsionen des Larynx im Behandlungsstuhl unter Sprühbetäubung und ohne Sedierung ist weltweit etabliert [32]. Zum Einsatz kommen fasergeführter Laser wie beispielsweise Diodenlaser [33], der bereits erwähnte KTP-Laser und neuerdings auch der sog. „blue Iaser" [23]. Dieser neue Laser mit einer Wellenlänge von $445 \mathrm{~nm}$ ist dem KTP-Laser vom Absorptionsspektrum her weitgehend vergleichbar. Gegenwärtig wird der differenzielle Nutzen dieses Lasers noch untersucht, allerdings scheinen mit diesem Laser photoangiolytische und schneidende Effekte kombiniert zur Anwendung zu kommen [23].

Durch die dünnen Glasfasern ist v. a. die transnasale Behandlung mit flexiblen Optiken möglich. Bei dysplastischem Gewebe mit sichtbarer deutlicher Angiogenese im Sinne von ICPL kann durch die selektive Photoangiolyse eine Behandlung a priori oder eine Nachbehandlung erfolgen [34, 35].

Auch zur schonenden Ablation von Stimmlippenpolypen wurden KTP-Laser bereits erfolgreich „office-based" eingesetzt [36]. Beim Verdacht auf das Vorliegen von Invasivität bleibt aber die Stützmikrolaryngoskopie mit histologischer Abklärung indiziert.

In der Abb. 6 wird ein routinemäßiger Lasereinsatz im Behandlungsstuhl ohne Sedierung bei einem 35-jährigen Patienten mit Larynxpapillomatose gezeigt. Der gepulste Laser ermöglicht die schmerzfreie Koagulation eines Papillomherds mit einer $400 \mu \mathrm{m}$ dünnen Glasfaser.

\section{Fazit für die Praxis}

- Strukturschonende angiolytische Lasertechniken, wie Kalium-Titanyl-Phosphat(KTP)-Laser und "blue laser", sind gegenwärtig bei vielen gutartigen Schleimhautveränderungen am Larynx einsetzbar und resultieren in guten postinterventionellen Stimmresultaten.

- Angiolytische Laser bieten darüber hinaus Möglichkeiten zur ambulanten Behandlung in Lokalanästhesie bei ausgewählten Patientenkollektiven.

- Insbesondere bei der juvenilen Larynxpapillomatose überwiegen zahlreiche Vorteile gegenüber herkömmlichen $\mathrm{CO}_{2}$-Laser-Techniken.
- Bei Schleimhautdysplasien und Larynxkarzinomfrühformen (T1a) kann die Behandlung mit angiolytischen Lasern zu einer verbesserten Stimmfunktion im Vergleich zum präoperativen Vorbefund und zu den zu erwartenden Resultaten bei $\mathrm{CO}_{2}$-Laser-Anwendung führen. Eine Kontrollmikrolaryngoskopie zum Ausschluss eines Tumorresiduums nach 4-6 Wochen bleibt obligat.

- Die lokoregionäre Kontrolle und damit die onkologische Sicherheit der transoralen Lasermikrochirurgie mittels Kalium-Titanyl-Phosphat-Laser (TLM-KTP) bei größeren Larynxkarzinomen (ab T2) muss erst in randomisierten und verblindeten Multizenterstudien weiter evaluiert werden.

\section{Korrespondenzadresse}

\section{S. Strieth}

Hals-Nasen-Ohren-Klinik, Universitätsmedizin Mainz

Langenbeckstr. 1, 55131 Mainz, Deutschland

sebastian.strieth@unimedizin-mainz.de

\section{Einhaltung ethischer Richtlinien}

Interessenkonflikt. Gemäß den Richtlinien des Springer Medizin Verlags werden Autoren und Wissenschaftliche Leitung im Rahmen der Manuskripterstellung und Manuskriptfreigabe aufgefordert, eine vollständige Erklärung zu ihren finanziellen und nichtfinanziellen Interessen abzugeben.

Autoren. M. Hess: A. Finanzielle Interessen: Fa. ARC Laser: Vortragshonorar, Reisekostenerstattung, Gerätestellung |Fa. Biolitec: Gerätestellung|Fa. Lumenis: Vortrags honorar, Reisekostenerstattung. - B. Nichtfinanzielle Interessen: Niedergelassener Phoniater und HNO-Arzt, Deutsche Stimmklinik, Hamburg |Akademisch affiliiert mit UKE (Univ.-Prof. am Universitätsklinikum Hamburg-Eppendorf)|Präsident PEVOC (Pan European Voice Conferences) | Past Präsident CoMeT (Collegium Medicorum Theatri)|Secretary General IAP (International Association of Phonosurgery) | Mitgliedschaften: DGPP (Deutsche Gesellschaft für Phoniatrie und Pädaudiologie), Deutsche Gesellschaft für HNO-Heilkunde, Kopf- und Halschirurgie, Executive Board EAV (European Academy of Voice), Deutsch-vietnamesische Gesellschaft zur Förderung der Medizin in Vietnam e. V., Berufsverband der Phoniater und Pädaudiologen (BVPP).

S. Strieth: A. Finanzielle Interessen: Deutsche Forschungsgemeinschaft (DFG), Bonn | Stiftung Tumorforschung Kopf-Hals, Wiesbaden |MED-EL AG, Innsbruck|Auris Medical, Basel| Merck Serono, Darmstadt | Otonomy, Inc., San Diego (USA) | Nordmark Arzneimittel, Uetersen | Andreas Fahl Medizintechnik-Vertrieb, Köln | Atos Medical, Troisdorf|Tracoe Medical, Nieder-Olm | Heimomed Heinze, Kerpen |Bromepithetik, Heidelberg | Fresenius Kabi, Bad Hersfeld. - MED-EL AG, Innsbruck|Sonofi Genzyme, Berlin | ALK-Abelló Arzneimittel, Hamburg. - Bezahlter Berater/interner Schulungsreferent/Gehaltsempfänger o. Ä.: Sanofi Genzyme, Berlin. - B. Nichtfinanzielle Interessen: Leitender Oberarzt, stellvertretender Klinikdirektor, Klinik für Hals-Nasen-Ohren-Heilkunde der Universitätsmedizin Mainz |Mitgliedschaften: Deutsche Gesellschaft für Hals-Nasen-Ohren-Heilkunde, Kopf- und Hals-Chirurgie |(DGHNO): Arbeitsgemeinschaft Onkologie, Arbeitsgemeinschaft für Plastische, Rekonstruktive und Ästhetische Kopf-Hals-Chirurgie (APKO), Arbeitsgemeinschaft deutschsprachiger Audiologen und Neurootologen (ADANO) | Deutsche Krebsgesellschaft (DKG): Interdisziplinäre Arbeitsgemeinschaft Kopf-Hals-Tumoren (IAG-KHT) |European Head and Neck Society (EHNS), Deutsche Gesellschaft für Allergologie und klinische Immunologie e. V. (DGAKI), Deutsche Gesellschaft für Ultraschall in der Medizin (DEGUM): StufeIII-Kursleiter, seit Februar 2015 stellvertretender Vorsitzender der Sektion Kopf-Hals | Deutsche Gesellschaft für Audiologie (DGA), Vereinigung Südwestdeutscher HalsNasen-Ohrenärzte, Deutscher Hochschulverband (DHV).

J. Hagemann: A. Finanzielle Interessen: J. Hagemann gibt an, dass kein finanzieller Interessenkonflikt besteht. - B. Nichtfinanzielle Interessen: Hals-, Nasen-, Ohrenklinik und Poliklinik, Plastische Operationen, Universitätsmedizin Mainz | Mitglied in folgenden Fachgesellschaften: Deutsche Gesellschaft für Hals-Nasen-Ohren-Heilkunde, 
Kopf-Hals-Chirurgie (DGHNOKHC), American Academy of Otolaryngology, Head and Neck Surgery, European Rhinologic Society.

Wissenschaftliche Leitung. Die vollständige Erklärung zum Interessenkonflikt der Wissenschaftlichen Leitung finden Sie am Kurs der zertifizierten Fortbildung auf www. springermedizin.de/cme.

Der Verlag erklärt, dass für die Publikation dieser CME-Fortbildung keine Sponsorengelder an den Verlag fließen.

Für diesen Beitrag wurden von den Autoren keine Studien an Menschen oder Tieren durchgeführt. Für die aufgeführten Studien gelten die jeweils dort angegebenen ethischen Richtlinien.

Open Access. Dieser Artikel wird unter der Creative Commons Namensnennung 4.0 International Lizenz veröffentlicht, welche die Nutzung, Vervielfältigung, Bearbeitung Verbreitung und Wiedergabe in jeglichem Medium und Format erlaubt, sofern Sie den/die ursprünglichen Autor(en) und die Quelle ordnungsgemäß nennen, einen Link zur Creative Commons Lizenz beifügen und angeben, ob Änderungen vorgenommen wurden.

Die in diesem Artikel enthaltenen Bilder und sonstiges Drittmaterial unterliegen ebenfalls der genannten Creative Commons Lizenz, sofern sich aus der Abbildungslegende nichts anderes ergibt. Sofern das betreffende Material nicht unter der genannten Creative Commons Lizenz steht und die betreffende Handlung nicht nach gesetzlichen Vorschriften erlaubt ist, ist für die oben aufgeführten Weiterverwendungen des Materials die Einwilligung des jeweiligen Rechteinhabers einzuholen.

Weitere Details zur Lizenz entnehmen Sie bitte der Lizenzinformation auf http:// creativecommons.org/licenses/by/4.0/deed.de.

\section{Literatur}

1. Vaughan CW, Strong MS, Jako GJ (1978) Laryngeal carcinoma-transoral treatment utilizing CO2-laser. Am J Surg 136:490-493

2. Steiner W (1993) Results of curative laser microsurgery of laryngeal carcinomas. Am JOtolaryngol 14:116-121

3. Sjogren EV, Van Rossum MA, Langeveld TPM et al (2008) Voice outcome in Tla midcord glottic carcinoma. Arch Otolaryngol 134:965-972

4. Folkman J (1971) Tumor angiogenesis: therapeutic implications. N Engl J Med 285:1182-1186. https://doi.org/10.1056/NEJM197111182852108

5. Piawah S, Venook AP (2019) Targeted therapy for colorectal cancer metastases: a review of current methods of molecularly targeted therapy and the use of tumor biomarkers in the treatment of metastatic colorectal cancer. Cancer 125(23):4139-4147. https://doi.org/10.1002/cncr.32163

6. Zeitels SM, Burns JA, Lopez-Guerra Getal (2008) Photoangiolytic laser treatment of early glottic cancer: a new management strategy. Ann Otol Rhinol Laryngol Suppl 199:3-24

7. Murono S, Endo K, Kondo S et al (2013) Oncological and functional outcome after transoral 532-nm pulsed potassium-titanyl-phosphate laser surgery for T1 a glottic carcinoma. Lasers Med Sci 28:615-619

8. Nouraei Sa R, Dorman EB, Macann A et al (2019) Outcomes of treating early glottic neoplasms with a potassium Titanyl phosphate laser. Ann Otol Rhinol Laryngol 128:85-95

9. Strieth S, Ernst BP, Both l et al (2019) Randomized controlled single-blinded clinical trial of functional voice outcome after vascular targeting KTP laser microsurgery of early laryngeal cancer. Head Neck 41:899-907

10. Zeitels SM, Burns JA (2014) Oncologic efficacy of angiolytic KTP laser treatment of early glottic cancer. Ann Otol Rhinol Laryngol 123:840-846

11. American Academy of Otolaryngology - Head and Neck Surgery (2014) American academy of otolaryngology — head and necksurgery. Position statement: in-office photoangiolytic laser treatment of laryngeal pathology. http://www.entnet.org. Accessed 1 Oct 2019

12. Havens FZ (1953) Bilateral paralysis of the vocal cords: treatment by transoral arytenoidectomy. Laryngoscope 63:475-484

13. Vaughan CW (1978) Transoral laryngeal surgery using the CO2 laser: laboratory experiments and clinical experience. Laryngoscope 88:1399-1420

14. AWMF(2019) Leitlinienprogramm Onkologie:Diagnostik, Therapie und Nachsorge des Larynxkarzinoms, S3-Leitlinie. Langversion 1.0

15. Sittel C, Eckel HE, Eschenburg C (1998) Phonatory results after laser surgery for glottic carcinoma. Otolaryngol Head Neck Surg 119:418-424

16. Keilmann A, NapiontekU, Engel C et al (2011) Long-term functional outcome after unilateral cordectomy. ORL J Otorhinolaryngol Relat Spec 73:38-46
17. Remacle M, Eckel HE, Antonelli A et al (2000) Endoscopic cordectomy. A proposal for a classification by the Working Committee, European Laryngological Society. Eur Arch Otorhinolaryngol 257:227-231.https://doi.org/10.1007/s004050050228

18. Abdurehim Y, HuaZ, Yasin Yetal (2012) Transoral laser surgery versus radiotherapy: systematic review and meta-analysis for treatment options of T1a glottic cancer. Head Neck34:23-33. https://doi.org/10.1002/hed.21686

19. Greulich MT, Parker NP, LeeP et al (2015) Voice outcomes following radiation versus laser microsurgery for 11 glottic carcinoma: systematic review and meta-analysis. Otolaryngol Head Neck Surg 152:811-819

20. Robert A, Pointreau $Y$, Janoray $G$ et al (2017) A large French multicenter retrospective series of T1-T2N0 vocal cords carcinomas treated with exclusive irradiation. Cancer Radiother 21:286-290

21. Broadhurst MS, Akst LM, Burns JA et al (2007) Effects of $532 \mathrm{~nm}$ pulsed-KTP laser parameters on vessel ablation in the avian chorioallantoicmembrane: implications forvocal fold mucosa. Laryngoscope 117:220-225

22. Burns JA, Zeitels SM, Akst LM et al (2007) $532 \mathrm{~nm}$ pulsed potassium-titanylphosphate laser treatment of laryngeal papillomatosis under general anesthesia. Laryngoscope 117:1500-1504

23. Hess MM, Fleischer S, Ernstberger M (2018) New $445 \mathrm{~nm}$ Blue laser for laryngeal surgery combines photoangiolytic and cutting properties. Eur Arch Otorhinolaryngol 275:1557-1567

24. Mallur PS, Johns MM 3rd, Amin MR et al (2014) Proposed classification system for reporting 532-nm pulsed potassium titanyl phosphate laser treatment effects on vocal fold lesions. Laryngoscope 124:1170-1175

25. Young VN, Mallur PS, Wong AW et al (2015) Analysis of potassium titanyl phosphate laser settings and voice outcomes in the treatment of Reinke's edema. Ann Otol Rhinol Laryngol 124:216-220

26. Nawka T, Wiesmann U, Gonnermann U (2003) Validation of the German version of the Voice Handicap Index. HNO 51(11):921-930. https://doi.org/10.1007/s00106003-0909-8

27. Rosen CA, Murry T, Zinn A et al (2000) Voice handicap index change following treatment of voice disorders. JVoice 14:619-623

28. Van Loon Y, Sjogren EV, Langeveld TP et al (2012) Functional outcomes after radiotherapy or laser surgery in early glottic carcinoma: a systematic review. Head Neck34:1179-1189

29. Zeitels SM, Akst LM, Burns JA et al (2006) Office-based 532-nm pulsed KTP laser treatment of glottal papillomatosis and dysplasia. Ann Otol Rhinol Laryngol 115:679-685. https://doi.org/10.1177/000348940611500905

30. Zeitels SM, Lopez-Guerra G, Burns JA et al (2009) Microlaryngoscopic and officebased injection of bevacizumab (Avastin) to enhance 532-nm pulsed KTP laser treatment of glottal papillomatosis. Ann Otol Rhinol Laryngol Suppl 201:1-13

31. Zeitels SM, Barbu AM, Landau-Zemer T et al (2011) Local injection of bevacizumab (Avastin) and angiolytic KTP laser treatment of recurrent respiratory papillomatosis of the vocal folds: a prospective study. Ann Otol Rhinol Laryngol 120:627-634

32. Zeitels SM, Burns JA (2007) Office-based laryngeal laser surgery with the 532-nm pulsed-potassium-titanyl-phosphate laser.Curr Opin Otolaryngol Head Neck Surg 15:394-400. https://doi.org/10.1097/MOO.0b13e3282f1fbb2

33. Janda P, Leunig A, Sroka R et al (2004) Preliminary report of endolaryngeal and endotracheal laser surgery of juvenile-onset recurrent respiratory papillomatosis by Nd:YAG laser and a new fiber guidance instrument. Otolaryngol Head Neck Surg 131:44-49. https://doi.org/10.1016/j.otohns.2003.1011.1019

34. Hess MFS (2018) Photoangiolytische Laser in der Laryngologie. Sprache Stimme Gehör 42:185-191

35. Zeitels SM (2015) Transoral and transcervical surgical innovations in the treatment of glottic cancer. Otolaryngol Clin North Am 48:677-685. https://doi.org/10.1016/ j.otc.2015.04.012

36. Sridharan S, Achlatis S, Ruiz R et al (2014) Patient-based outcomes of in-office KTP ablation of vocal fold polyps. Laryngoscope 124:1176-1179 
? Die weitere Verbreitung und Durchsetzung der transoralen Lasermikrochirurgie in der Onkologie ist begründet durch ...

O eine geringe Komplikationsrate durch Nachblutungen.

O fehlende atemwegskompromittierende Komplikationen.

○ eine gegenüber der offenen Chirurgie verbesserte onkologische Resektion.

○ ein verbessertes Gesamtüberleben im Vergleich zur primären Radio(chemo)therapie nach Behandlung kleiner Tumoren.

○ ausgeprägte Laryngeal-Framework-Effekte.

? Im Gegensatz zum $\mathrm{CO}_{2}$-Laser bieten photoangiolytische Laser ...

O tief einschneidende Effekte am Gewebe.

O eine weitgehende Schonung der schwingungsähigen Lamina propria.

○ keine thermischen Schäden am Kehlkopfgewebe.

O neue Möglichkeiten des Organerhalts bei fortgeschrittenen Karzinomen.

○ Nachteile in der Handhabung.

? In der aktuell gültigen S3-Leitlinie zu Diagnostik, Therapie und Nachsorge des Larynxkarzinoms ...

O finden die photoangiolytischen Laserverfahren Erwähnung als neuer Standard bei der Therapie von Frühformen.

O sind lediglich $\mathrm{CO}_{2}$-Laser zur transoralen Lasermikrochirurgie (TLM) empfohlen.
O hat die transorale Lasermikrochirurgie einen hohen Stellenwert für die Behandlung von glottischen T1- und T2-Karzinomen.

O wird die primäre Radiotherapie wegen eines besseren Stimmresultats bei T1aTumoren empfohlen.

○ ist die logopädische Stimmtherapie nach Therapie obligat.

? Ein 46-jähriger Patient stellt sich in der phoniatrischen Sprechstunde nach erfolgter transoraler Laseroperation eines T1b-Glottiskarzinoms vor. Zur Bewertung des funktionellen Ergebnisses ...

O hat sich der Voice-Handicap-Index(VHI)Fragebogen zur subjektiven Bewertung der Beeinträchtigung der Stimmfunktion in zahlreichen Studien bewährt.

○ ist eine Videostroboskopie zur subjektiven Untersuchung der Schwingungsfähigkeit ausreichend.

muss eine Lungenfunktionsprüfung erfolgen.

O stellt die GRBAS-Systematik - Grade/ Grad, Roughness/Rauheit, Breathiness/ Behauchtheit, Asthenia/Asthenie (Klangarmut), Strain/Pressen - eine subjektive Beurteilung durch die Patienten dar.

O sind die objektiv messbaren Parameter entscheidend. ?u den aktuellen Prinzipien bei transoralen Laserresektionen von ausgedehnten Malignomen am Larynx mit dem $\mathrm{CO}_{2}$-Laser gehören ...

0 ein Sicherheitsabstand von $5 \mathrm{~mm}$.

$\bigcirc$ das Ziel, eine Tracheotomie zu vermeiden.

$\mathrm{O}$ das Piecemeal-Vorgehen.

O die Verwendung der Klassifizierung nach Mallur.

O eine bessere Stimmfunktion als bei Bestrahlung.

? Bei transoralen direkten Kalium-Titanyl-Phosphat(KTP)-Laser-Behandlungen am Larynx ...

○ existiert eine Chordektomieklassifizierung der European Laryngological Society.

O versteht man unter KTP-V-Resektionen ein Debulking des Tumors.

O sollte man nicht auf angiogenetische torquierte Gefäße (ICPL) achten.

o sind wiederholte Anwendungen möglich.

$\mathrm{O}$ ist eine Stützlaryngoskopie erforderlich.

? Die Möglichkeiten zur Gewebeschonung durch photoangiolytische Laserverfahren ergeben sich ...

O durch komplette Energieabsorption an der Epitheloberfläche.

$\bigcirc$ durch selektive Absorption der Energie von Hämoglobin und Obliteration kleiner Gefäße.

O durch einen größeren Arbeitsabstand im Vergleich zum $\mathrm{CO}_{2}$-Laser.

\section{Informationen zur zertifizierten Fortbildung}

Diese Fortbildung wurde von der Ärztekammer Nordrhein für das „Fortbildungszertifikat der Ärztekammer" gemäß 55 ihrer Fortbildungsordnung mit 3 Punkten (Kategorie D) anerkannt und ist damit auch für andere Ärztekammern anerkennungsfähig.
Anerkennung in Österreich: Für das Diplom-Fortbildungs-Programm (DFP) werden die von deutschen Landesärztekammern anerkannten Fortbildungspunkte aufgrund der Gleichwertigkeit im gleichen Umfang als DFP-Punkte anerkannt (§ 14, Abschnitt 1 , Verordnung über ärztliche Fortbildung, Österreichische Ärztekammer (ÖÄK) 2013).
Hinweise zur Teilnahme:

- Die Teilnahme an dem zertifizierten Kurs ist nur online auf www.springermedizin.de/cme möglich.

- Der Teilnahmezeitraum beträgt 12 Monate. Den Teilnahmeschluss finden Sie online beim Kurs.

- Die Fragen und ihre zugehörigen Antwortmöglichkeiten werden online in zufälliger Reihenfolge zusammengestellt.
- Pro Frage ist jeweils nur eine Antwort zutreffend.

- Für eine erfolgreiche Teilnahme müssen 70\% der Fragen richtig beantwortet werden.

- Teilnehmen können Abonnenten dieser Fachzeitschrift und e.Med- und e.Dent-Abonnenten. 
$\bigcirc$ durch höhere thermische Energieeinwirkung im Vergleich zum $\mathrm{CO}_{2}$-Laser.

O durch epitheliale Gewebeablation.

? In Bezug auf die aktuell eingesetzten Laser in der Laryngologie ist korrekt:

○ "Blue laser" wirken nicht angiolytisch.

O Kalium-Titanyl-Phosphat(KTP)-Laser-Anwendungen sind tief einschneidende Verfahren.

O Energie der Wellenlänge $532 \mathrm{~nm}$ wird hauptsächlich vom Epithel absorbiert.

O $\mathrm{CO}_{2}$-Laser sind der Goldstandard für die transorale Lasermikrochirurgie (TLM) bei fortgeschrittenen Tumoren.

O Die S3-Leitlinie zur Diagnose, Therapie und Nachsorge des Larynxkarzinoms schreibt explizit nur den Gebrauch des $\mathrm{CO}_{2}$-Lasers am Larynx vor
? Ein 10-jähriges Mädchen wird Ihnen in der Hochschulambulanz zur Behandlung einer juvenilen Larynxpapillomatose vorgestellt. Welche der folgenden Aussagen sollten Sie für das Gespräch mit den Eltern am ehesten berücksichtigen?

O Eine einmalige Laserbehandlung ist meistens ausreichend.

O Das funktionelle Ergebnis ist nachrangig, da es sich um eine Präkanzerose handelt.

O Das photoangiolytische Verfahren bietet eine Chance zur Behandlung in örtlicher Betäubung.

O Die simultane Applikation von VascularEndothelial-Growth-Factor(VEGF)-Antikörpern ist nicht sinnvoll.

O Tracheotomien bieten mehr Sicherheit und werden daher empfohlen.
? Angiolytische Laserbehandlungen am wachen Patienten ...

O sollten nur im Rahmen von klinischen Studien vorgenommen werden.

○ werden in der Regel durch flexible Optiken ermöglicht.

o sind von Blue-Laser-Anwendungen abzugrenzen.

O können nicht ohne Sedierung vorgenommen werden.

○ kommen v. a. für größere Tumoren infrage.

Der Update Newsletter Innere Medizin liefert Ihnen regelmäßig Aktuelles und Wissenswertes aus allen medizinischen Fachgebieten:

- CME-Beiträge aus den Fachzeitschriften von Springer Medizin

- umfassende Übersichtsbeiträge und interessante Kasuistiken

- aktuelle internationale Studien

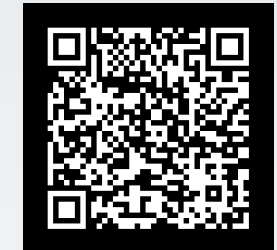

- Kongress-Highlights und Themen-Specials

- News aus Berufs- und Gesundheitspolitik

Jetzt Newsletter auswählen und kostenlos bestellen unter

www.springermedizin.de/mynewsletters 